\title{
Robust Noise Reduction and Echo Cancellation
}

\author{
Kristian Kroschel, Martin Heckmann \\ Universitaet Karlsruhe, Institut fuer Nachrichtentechnik, Institut fuer Automation und Robotik \\ Kaiserstrasse 12, D-76128 Karlsruhe \\ kroschel@int.uni-karlsruhe.de
}

Key words: Robustness, noise reduction, echo cancellation

Abstract: Mobile phones used in cars require for safety reasons handset-free operation. This implies that a front-end is used which is able to reduce the ambient noise so that an acceptable intelligiblity is guaranteed for the far-end customer. Furthermore, the acoustic echo transmitted from the loudspeaker to the microphone has to be reduced so that it is not audible.

Another aspect has to be taken into account: the system has to be robust, i.e. it has to be operable in a wide range of the signal-to-noise ratio down to $0 \mathrm{~dB}$ or less. Furthermore the demand for a-priori knowledge should be as low as possible because acquisition of knowledge induces time delays unacceptable for real-time systems and contradicts a low-cost solution which is required for a mass product.

New developments in this paper concern the segmentation of the frequency band into subbands for all relevant components: noise reduction, echo cancellation and double-talk detection. Furthermore new concepts for echo reduction are presented.

\section{INTRODUCTION}

Handset-free communication from a car is of signi $1 / 2$ cant interest: the main reason is safety since the driver should have all the time the ability to control the car manually which is not the case if he holds a mobile phone. Furthermore comfort is another aspect to use handset-free phones.

There are a couple of these phones on the market which are adapted to the special conditions in the car but still there is need for further research because the quality of the processed speech deserves further improvement 
and some of the commercially available systems degrade significantly in low signal-to-noise environments.

Two goals have to be met by handset-free phones: the first is to reduce the influence of the environmental noise so that the intelligibility of the transmitted speech is improved, the second is to reduce the speech echo from the far-end user which is generated on the path from the loudspeaker to the microphone.

In this paper robustness is the main issue of discussion. By robustness we understand that both operations, the noise reduction and the echo cancellation are as much as possible independent of the input signalto-noise ratio, i.e they render an appropriate result and are stable even at low signal-to-noise ratios and require as few a-priori information as possible.

\section{NOISE REDUCTION}

There are many approaches for noise reduction. The most advanced ones are those based on signal estimation using Kalman filters [9]. This solution requires an appropriate model of speech and noise, respectively, which is in contradiction to our demand for a design which does not need much a-priori knowledge.

Since all model based systems require a lot of a-priori knowledge they all are discarded from our list of candidates for noise reduction. Thus we have to look for black-box solutions such as singular value decomposition, Karhunen Loève transform, vector quantization and others. They need much less a-priori information but the effort for mathematical computations is quite high since they need the calculation and inversion of correlation matrices. Furthermore, the quality of the processed speech might be degraded significantly since the parameters representing the uncorrupted speech are manipulated severely in those regions in which the parameters of the noise are strong. There are some ideas to overcome this problem which try to extract the lost information from the remaining parameters but this is an operation which requires a high effort.

A well-known solution for noise reduction, which is simple to implement, which is more or less independent of the input signal-to-noise ratio, and which does not depend too much on a-priori knowledge is based on Wiener filters [9] and known as spectral subtraction [14]:

$$
H\left(e^{j \Omega}\right)=\frac{S_{S S}\left(e^{j \Omega}\right)}{S_{R R}\left(e^{j \Omega}\right)}=\frac{S_{R R}\left(e^{j \Omega}\right)-S_{N N}\left(e^{j \Omega}\right)}{S_{R R}\left(e^{j \Omega}\right)}
$$




$$
\approx \begin{cases}1-a \cdot \frac{S_{N N}\left(e^{j \Omega}\right)}{S_{R R}\left(e^{j n}\right)} & 1-a \cdot \frac{S_{N N}\left(e^{j \Omega}\right)}{S_{R R}\left(e^{j n}\right)} \geq b \\ b & 1-a \cdot \frac{S_{N N}\left(e^{j \Omega}\right)}{S_{R R}\left(e^{j n}\right)}<b\end{cases}
$$

with $S_{S S}\left(e^{j \Omega}\right), S_{R R}\left(e^{j \Omega}\right)$ and $S_{N N}\left(e^{j \Omega}\right)$ the power density spectra of the clean speech $s(k)$ and the speech corrupted by noise $r(k)$ and the noise $n(k)$, respectively. A block diagram of this system is given in Figure 1.

The a-priori knowledge required for this approach is the estimate of the noise power $S_{N N}\left(e^{j \Omega}\right)$. This can be updated in speech pauses

$$
\hat{S}_{N N}\left(e^{j \Omega}, i\right)=\alpha \cdot \hat{S}_{N N}\left(e^{j \Omega}, i-1\right)+(1-\alpha) \cdot\left|N\left(e^{j \Omega}, i\right)\right|^{2}
$$

with $N\left(e^{j \Omega}, i\right)$ the spectrum of the noise $n(k)$ in the speech pause with index $i$. The drawback of this approach is that highly instationary noise will be estimated quite unsatisfactorily. Furthermore, some time will pass by until the first speech pause is detected and an estimate of the noise is available. The requirement of a noise activity detector (VAD) can be counted under the negative aspects, too. There are two solutions without the need of a VAD: first, a microphone array might be used with the output of an estimate of the corrupted speech signal and of an estimate of the corrupting noise [7]. If this is assumed to be too expensive a simpler solution uses so-called minimum statistics [10], which means that an estimate of the noise power is permanently calculated as the minimum of the power in a specified time interval. The set-up time of this approach has proven to be very short in practical applications.

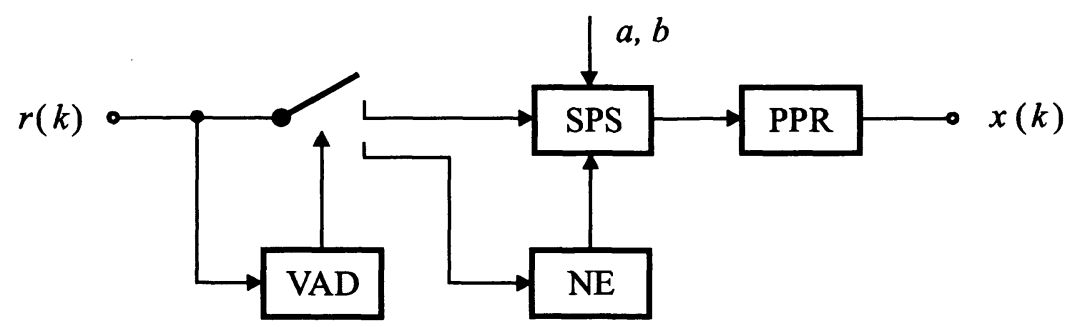

Figure 1: Noise reduction based on spectral subtraction (SPS). VAD: voice activity detector, NE: noise estimation, PPR: post processing.

The main drawback of spectral subtraction for noise reduction is that musical tones are produced which sound very unnatural and are very annoying. Spectral subtraction is parameterized by two values: the overestimate $a$ with typical values $1 \leq a \leq 3$ and the spectal floor $b$ with typical 
values $0.1 \leq b \leq 0.3$. With $b$ set close to the upper limit the musical tones are made less audible but on the other hand the noise suppression effect is reduced so that intelligibility is affected. Two methods are suggested in literature to reduce the influence of musical tones: post-processing of data with a median filter of a typical lenght of $L=3$ or $L=5$ [2], an alternative is to exploit psycho acoustics [3], [6]. To avoid musical tones, those isolated spectral lines are set to the spectral floor which pass the auditory threshold and are not masked by the neighbouring spectral lines of the speech singnal.

Further modifications of spectral subtraction include a frequency dependent overestimate $a(\Omega)$ parameter [11] and the segmentation of the frequency range into specific bands.

\section{ECHO CANCELLATION}

The most satisfying solution for echo cancellation is the canceller based on the least mean square (LMS) algorithm [1], [15]

$$
\mathbf{h}(k+1)=\mathbf{h}(k)+\mu(k) \cdot f(k) \cdot \mathbf{x}(k)
$$

with $\mathbf{h}(k)=(h(1), h(2), \ldots, h(L))^{T}$ the vector of the impulse response $h(k), 1 \leq k \leq L$ of the echo path, $f(k)=\hat{y}(k)-y(k)$ the error of the echo $y(k)$ and its estimate $\hat{y}(k), \mathbf{x}(k)=(x(k), x(k-1), \ldots, x(k-L+1))^{T}$ the input of the echo path and $0<\mu(k)<2 /|\mathbf{x}(k)|^{2}$ the adaptation constant. A block diagram of this algorithm is given in Figure 2.

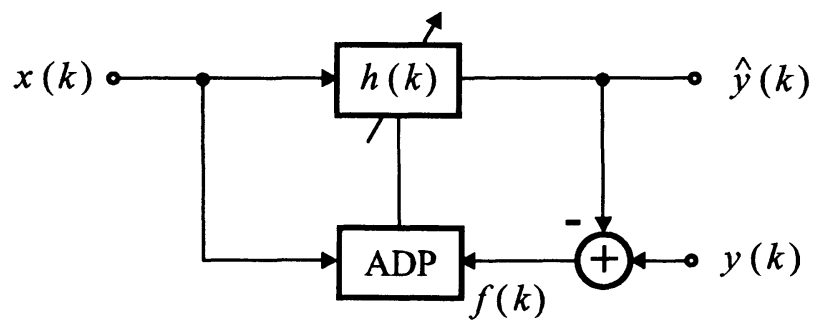

Figure 2: Least mean square estimation of impulse response $h(k)$. ADP: adaptive processing of parameters.

Since noise reduction is implemented in the frequency domain an algorithm for echo cancellation formulated in the frequency domain is 
preferred. In this case the signals are cut into blocks of lenght $B$. The result is the frequency least mean square (FLMS) algorithm [13]

$$
\mathbf{H}(j+1)=\mathbf{H}(j)+\mu(j) \cdot \nabla(j)
$$

with $\mathbf{H}$ the FFT of the augmented vector $\mathbf{h}(k), \nabla(j)=g\left(\mathbf{X}^{*}(j) * \mathbf{F}(j)\right)$ the gradient of the error $\mathbf{F}(j)$ which is the FFT of the augmented vector $\mathbf{f}(j)=(f(j B), f(j B-1), \ldots, f(j B-L+1))^{T}$ and $\mathbf{X}$ the FFT of the augmented vector $\mathbf{x}(k)$. Augmentation is required to avoid aliasing by applying overlad-add for the convolution.

These algorithms do not require a high computational load - e.g. no matrix inversion as the recursive least square (RLS) algorithm - and their adaptation to changes in the acoustical environment is fast enough for practical applications. The FLMS algorithm has the further advantage that the input values are more or less uncorrelated which improves the adaptation behaviour.

Due to changes of the acoustic transmission path between the loudspeaker and the microphone caused by movements of persons within the path or changes of the environment - opening and closing of windows and doors etc. - the echo canceller has to be adaptive. Speed and accuracy of the adaptation depend on the update parameter $\mu$ which is sensitive with respect to the signal-to-noise ratio and which is calculated for each frequency band separately [16].

To demonstrate the robustness of this approach, Figure 3 shows the behaviour of adaptaion in an adverse environment: with a low signalto-noise ratio - $\mathrm{SNR}=0 \mathrm{~dB}$ - and a high influence of double talk - the power ratio of the echo and the local speech is $0 \mathrm{~dB}$ - the adaptaion of the frequency selective echo cancellation is superior to the conventional approach with an adaptation over the full spectral band. The compensation of the echo is expressed as echo return loss enhancement (ERLE) which fluctuates significantly due to the local speech. For comparison of the new and conventional approach, the system distance between the transfer function of the transmission path from the loudspeaker to the microphone and the adaptive echo canceller are given.

Noise is in this case the speech signal of the local speaker and the environmental noise. Adaptation of the echo canceller is executed only in periods in which the far-end speaker is active, i.e. an echo is generated. Therefore a voice activity detector is required in the loudspeaker path. 

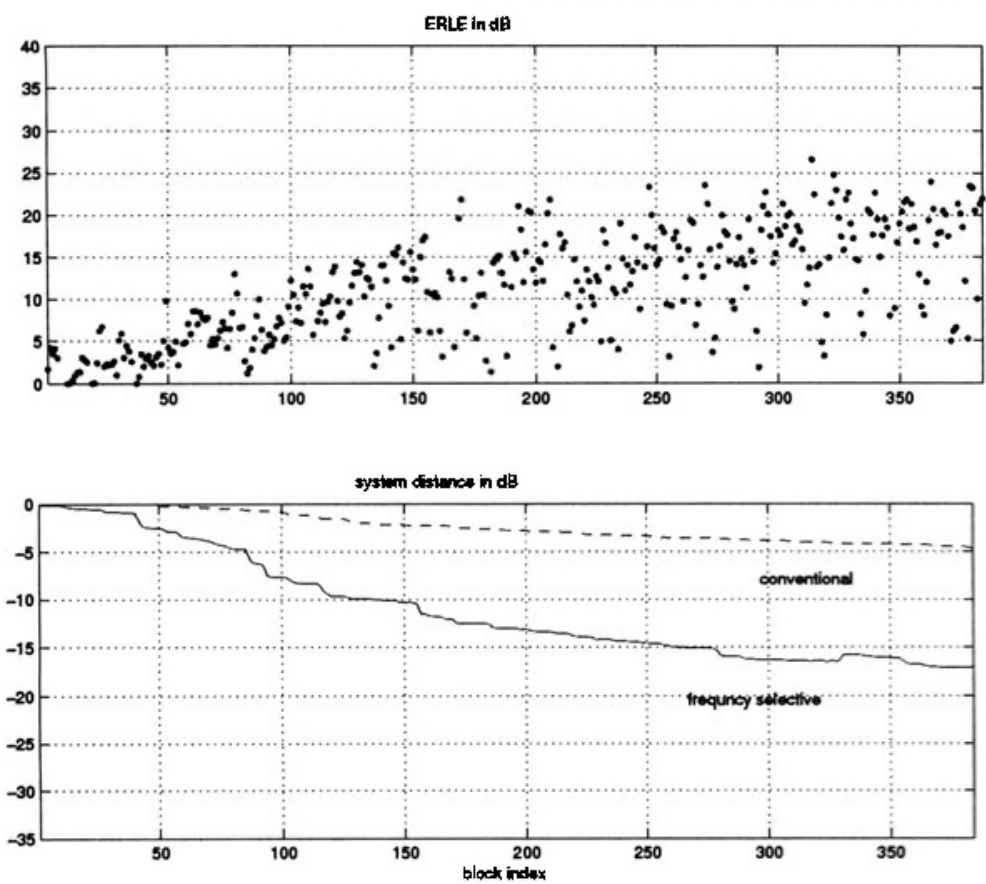

Figure 3: Echo cancellation in an adverse environment: $\mathrm{SNR}=0 \mathrm{~dB}$, power ratio of echo and local speech $0 \mathrm{~dB}$.

The problem with the LMS and FLMS algorithms is that they rarely achieve the required attenuation of the echo specified by a given ERLE value. Therefore additional measures have to be taken. The one which will be discussed here further is the application of the so-called frequency selective gain control (FSGC) which allocates a gain value $H_{i}\left(e^{j \Omega}\right)$ to the two transmission paths from the local speaker to the remote speaker and vice versa. The gain is frequency dependent and controlled by the magnitude of the speech spectra in the two transmission paths:

$$
\begin{aligned}
H_{l}\left(e^{j \Omega}\right) & = \begin{cases}\beta_{l} \mid \frac{S_{l}\left(e^{j \Omega}\right) \mid}{\left|S_{f}\left(e^{j n}\right)\right|} & \beta_{l} \frac{\mid S_{l}\left(e^{j \Omega} \mid\right.}{\left|S_{f}\left(e^{n}\right)\right|}<1 \\
1 & \text { elsewhere }\end{cases} \\
H_{f}\left(e^{j \Omega}\right) & = \begin{cases}\beta_{f} \mid \frac{\left|S_{f}\left(e^{j n}\right)\right|}{\left|S_{n}\left(e^{j n}\right)\right|} & \beta_{l} \mid \frac{S_{f}\left(e^{j n}\right) \mid}{S_{n}\left(e^{j n}\right) \mid}<1 \\
1 & \text { elsewhere }\end{cases}
\end{aligned}
$$

with $l$ for the local speaker and $f$ for the far-end speaker. According to Figure $3, H_{f}\left(e^{j \Omega}\right)$ is the transfer function of the far-end speech signal $x_{f}(k)$ to the output $y_{f}(k)$ of the local loudspeaker, e.g. $S_{i}\left(e^{j \Omega}\right)$ denotes 
the spectrum of the transmitted speech which will be calculated by FFT. Comparing the magnitudes of the spectra of the local and the remote speaker the frequency axis is separated into bands. These bands can be as narrow as one spectral bin of the FFT. The width of these bands depends on the following aspects: the quality of the processed speech signal, the residual noise and the effort required for implementation.

With this approach double-talk communication is possible in contrast to conventional gain controls which cut off either one or the other transmission path. The desired echo attenuation is achieved by an appropriate reduction of the gain in each spectral band. This frequency dependent attenuation distorts the speech signal. But this distortion is almost not audible since those spectral components which are cut away from the spectrum of the local speaker are replaced by the corresponding spectral components of the far-end speaker. This is a very robust approach with the drawback that the speech signal is degraded for those listening to the dialogue of the local and the far-end speaker.

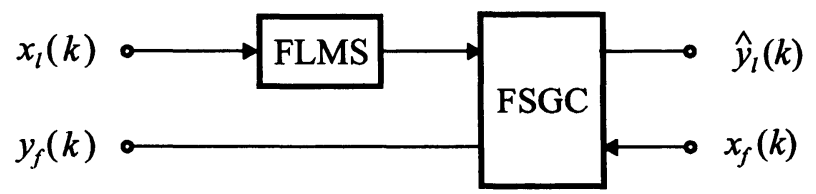

Figure 4: Combined echo cancellation. FLMS: frequency least mean square algorithm, FSGC: frequency selective gain control.

For the design of a robust echo canceller both approaches, echo compensation and frequency selective gain control, are combined. The idea is to exploit the absence of speech distortion of echo compensation for high signal-to-noise ratios and to use frequency selective gain control for low signal-to-noise ratios. Both components either can be arranged in parallel or serially. The parallel structure exploits the advantage that the speech signal might not pass the frequency selective gain control at high signal-to-noise ratios so that the speech signal is not distorted. The problem is to fuse the estimates of the echo gained from both systems. The fusion is based on a measure of quality of the estimated echo, and the overall estimate is the weighted sum of both individual estimates.

A more sophisticated approach subdivides the full frequency range into frequency bands and fuses the individual bands according to the measure of quality. 
The cascade of the FLMS echo canceller and the frequency selective gain control FSGC given in Figure 4 is a simpler and robust solution: the FLMS component compensates the echo totally at high signal-to-noise ratios so that there is no need for further echo reduction by the subsequent FSGC. In this case $\hat{y}_{l}(k)$ is identical with the local speech. Thanks to the frequency selective adaptation of the FLMS even for low signal-tonoise ratios a significant contribution to echo reduction is gained. This is true even if the resulting ERLE gained by the FLMS is not sufficient. An example is given in the left part of Figure 5 which shows the echo reduction expressed in ERLE as a function of the data block index. It can be read from this result that some 150 data blocks are required for adaptation. The gained ERLE value of about $10 \mathrm{~dB}$ is not very satisfactory. The reason is the environmental noise with a signal-to-noise ratio of $\mathrm{SNR}=10 \mathrm{~dB}$. The noise has been picked up in a car and is slightly instationary due to changes of speed.
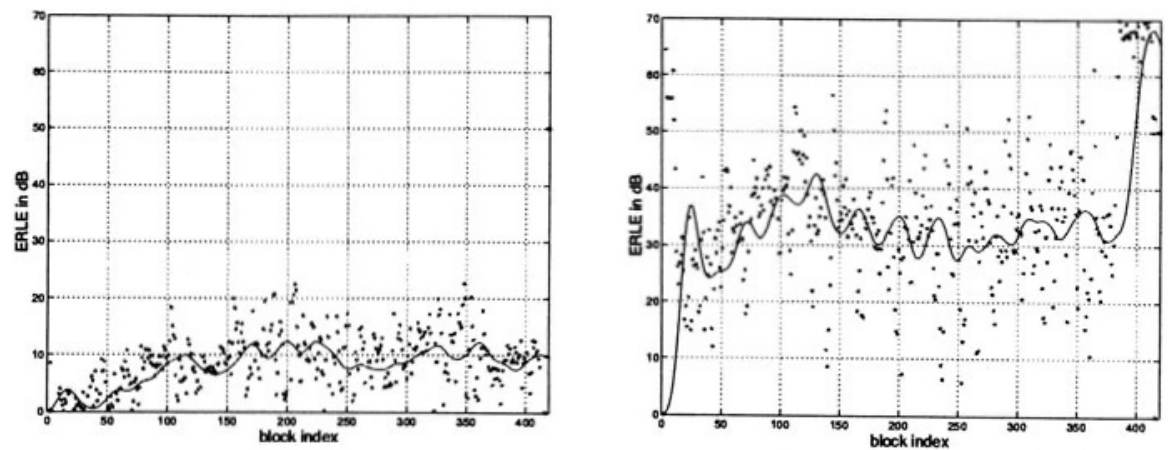

Figure 5: Echo cancellation: left by FLMS, right by the combination of FLMS and FSGC. Signal-to-noise ratio $S N R=10 \mathrm{~dB}$, power ratio of echo and local speech $0 \mathrm{~dB}$. ERLE in $\mathrm{dB}$ as a function of the processed data block.

The subsequent FSGC reduces the echo signifcantly as shown in the right part of Figure 5. The ERLE value is in the range of 30 to $40 \mathrm{~dB}$ which meets the requirements for practical application. In contrast to the FLMS algorithm the FSGC does not need any time for adaptation but renders almost from the start the required attenuation of the echo. The price for this operation is however an increase in distortion of both speech signals, the one transmitted to the far-end customer and the other one received locally. 


\section{COMBINING NOISE REDUCTION AND ECHO CAN- CELLATION}

Integration of noise reduction and echo cancellation is a topic reviewed earlier in literature [5], and can further be combined with source coding [4], [8]. The latter is of no concern in this paper. Either both operations are executed in the time or the frequency domain. Since spectral subtraction and the frequency selective gain control require a realization in the frequency domain, echo compensation is realized on the basis of the FLMS algorithm to reduce implementational load.

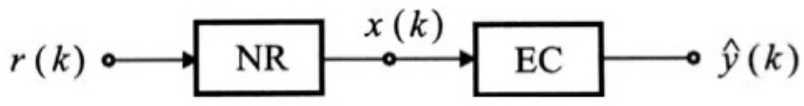

Figure 6: Combined noise reduction (NR) and echo cancellation (EC).

The question is how to integrate noise reduction and echo cancellation. A detailed investigation can be found in [12] where the question is answered whether noise reduction should precede echo cancellation or vice versa. Unfortunately a general answer cannot be given since it depends on the type of the applied noise reduction and echo cancellation method. Since noise reduction based on spectral subtraction is very robust we prefer the structure given in Figure 6 in which noise reduction is the first component followed by the echo canceller.

\section{CONCLUSION}

The system defined in this paper is an integration of well-known components. New developments are the segmentation of the frequency bands into subbands for all components: noise reduction, echo compensation together with the double-talk detector, and the frequency selective gain control. Furthermore, the combination of echo compensation with the frequency selective gain control and the interoperation of them are new ideas.

Tests with real-world signals have been executed for the LMS based, frequency selective echo canceller which showed that a significant gain in robustness in an adverse environment is gained. Informal listening tests have shown that the quality of the processed speech was appreciated by the listeners. 
Further research will be cncentrated on the integration of the frequency selective gain control to improve the measure of echo cencellation and the spectral subtraction for noise reduction, respectively.

\section{References}

[1] Cowan, C.F.N.; Grant, P.M.: Adaptive Filters. Prentice Hall, Englewood Cliffs, 1985

[2] Haulik, T.: Robuste Geraeuschreduktion fuer kompakte Mikrofonanordnungen. $\mathrm{PhD}$ dissertation, Karlsruhe 1988

[3] Haulik, T.; Linhard, K.; Schroegmeier, P: Residual Noise Suppression Using Psychoacoustic Criteria. Proc. EUROSPEECH 97

[4] Kroschel, K.; Barros, J.: Integrating Noise Suppression and Source Coding in Mobile Phones. Proc. Electronische Sprechsignalverarbeitung, Goerlitz, September 1999, pp. 96-103

[5] Kroschel, K.; Ihle, M.: Ein robustes System zum Freisprechen im Kraftfahrzeug. Proc. Electronische Sprechsignalverarbeitung, Cottbus, August 1997, pp. 78-84

[6] Kroschel, K.; Kujaw, K.: Speech Enhancement in a Noisy Environment Exploiting Phenomena of Hearing Physiology. Proc. 43rd Int. Scientific Colloquium, TU Ilmenau, September 1998, pp. 439-444

[7] Kroschel, K.; Lange, K.: Subband Array Processing for Speech Enhancement. Proc. EUROSPEECH-93, Berlin, September 1993, pp. 621-624

[8] Kuropatwinski, M.; Lekschat, D.; Kroschel, K.; Czyzewski, A.: Advanced Speech Signal Parameterization for Linear Predictive Coder Based Speech Enhancement, ISSEM'99, Gdansk, September 1999, pp. 43-51

[9] Kroschel, K.: Statistische Nachrichententheorie. 3rd ed, Springer, Berlin etc., 1996

[10] Martin, R.: An efficient algorithm to estimate the instantanous SNR of speech signals. Proc. EUROSPEECH-93, Berlin 1993, pp. 1093-1096

[11] Mekhaiel, M.: Untersuchung und Vergleich nichtlinearer Geraeuschreduktionsverfahren fuer robuste Spracherkennung. Diploma Thesis, Institut fuer Nachrichtentechnik, Universitaet Karlsruhe 1994

[12] Scalart, P.; Benamar, A.: A system for speech enhancement in the context of hands-free radiotelephony with combined noise reduction and acoustic echo cancellation. Speech Communication 20 (1992), pp. 203-214

[13] Soo, J.S.; Pang, K.K.: Multidelay block frequency domain adaptive filter. IEEE Trans. Acoustics, Speech and Signal Processing 38(2) (1990), pp. 373-376

[14] Vary, P.: On the Enhancement of Noisy Speech. Proc. EUSIPCO-83, Erlangen 1983, pp. 327

[15] Widrow, B.; Hoff Jr., M.E.: Adaptive switching circuits. IRE WESCON Conv. Rec. 1960,vol 4, pp. 96-104

[16] Heckmann, M.; Vogel, J; Kroschel, K.: Frequency selective step-size control for acoustic echo cancellation. Accepted for publication in Proc. EUSIPCO 2000, Sept. 4-8, 2000, Tampere, Finland 